\title{
Discrete Systolic Potentials During Ventricular Tachycardia in Patients with Prior Myocardial Infarction
}

\author{
FRANK BOGUN, M.D.,* BRADLEY KNIGHT, M.D., RAJIVA GOYAL, M.D., \\ GREGORY F. MICHAUD, M.D., S. ADAM STRICKBERGER, M.D., \\ STEFAN H. HOHNLOSER, M.D., * and FRED MORADY, M.D.
}

From the Division of Cardiology, Department of Internal Medicine, University of Michigan Medical Center, Ann Arbor, Michigan; and the *Division of Cardiology, J.W. Goethe University, Frankfurt, Germany

\begin{abstract}
Systolic Potentials. Introduction: Isolated diastolic potentials have been found to be helpful in identifying critical sites for ablation of ventricular tachycardia (VT) in patients with coronary artery disease. However, discrete potentials that occur during systole have not been previously described. The purpose of this study was to determine the significance of discrete systolic potentials during VT in patients with coronary artery disease.

Methods and Results: Twenty-seven patients with a mean age of $66 \pm 12$ years ( \pm standard deviation) who had coronary artery disease underwent radiofrequency catheter ablation of $\mathbf{4 2}$ VTs that had a mean cycle length of $\mathbf{4 8 6} \pm 78 \mathrm{msec}$. The only criterion used to select target sites for ablation was concealed entrainment, which was present at 92 sites. Thirty-five of the 42 VTs $(83 \%)$ were successfully ablated. A discrete systolic potential was recorded during 7 of the $\mathbf{4 2}$ VTs $(17 \%)$. In all cases, the interval between the discrete systolic potential and the next QRS complex was equal to the stimulus-QRS interval during concealed entrainment. At all seven sites where a discrete systolic potential was recorded, delivery of radiofrequency energy resulted in successful ablation of the VT.

Conclusion: Discrete systolic potentials may be present in patients with coronary artery disease in approximately $17 \%$ of VTs in which there is concealed entrainment. If the interval between the discrete systolic potential and the next QRS complex matches the stimulus-QRS interval during concealed entrainment, delivery of radiofrequency energy is likely to result in successful ablation of the VT. (J Cardiovasc Electrophysiol, Vol. 10, pp. 364-369, March 1999)
\end{abstract}

concealed entrainment, systolic potential, ventricular tachycardia

\section{Introduction}

Isolated diastolic potentials have been found to be helpful in localizing critical areas within the reentry circuit of ventricular tachycardia (VT) in patients with a history of myocardial infarction. ${ }^{1-9}$ However, discrete potentials during systole have not neen previously described. The purpose of this study was to determine the incidence of discrete systolic potentials during VT at sites of concealed entrainment in patients with

Address for correspondence: Fred Morady, M.D., University of Michigan Medical Center, 1500 East Medical Center Dr., UHB1F245, Ann Arbor, MI 48109-0022. Fax: 734-936-7026.

Manuscript received 17 September 1998; Accepted for publication 19 November 1998. prior myocardial infarction, and to assess their value in identifying effective target sites for ablation.

\section{Methods}

\section{Patient Characteristics}

The subjects of this study were 27 patients with coronary artery disease and prior myocardial infarction who were referred for radiofrequency catheter ablation of hemodynamically tolerated VT. There were 21 men and 6 women, their mean age being $66 \pm 12$ years $( \pm$ standard deviation). The mean left ventricular ejection fraction was $0.31 \pm 0.12$. Eighty-three different 
monomorphic VTs were induced in these 27 patients ( 1 to 5 per patient). A total of 42 VTs that had a mean cycle length of $486 \pm 78 \mathrm{msec}$ were targeted for ablation. Twenty-two of the VTs had a right bundle branch block configuration, while 20 had a left bundle branch block configuration. An internal cardioverter defibrillator was implanted before or shortly after the ablation procedure in 23 of the 27 patients. At the time of the procedure, 21 patients were being treated with amiodarone, 2 with sotalol, and 2 with a Class I antiarrhythmic drug. Fourteen of the 27 patients included in this study also were subjects of an earlier report. ${ }^{8}$

\section{Mapping Protocol}

The electrophysiology procedures were performed in the fasting state after informed consent was obtained. Midazolam and fentanyl were used for conscious sedation. An electrode catheter was inserted into a femoral vein and positioned in the right ventricle for programmed ventricular stimulation. Left ventricular mapping and ablation were performed with a 7-French, quadripolar electrode catheter that had an interelectrode spacing of 2-5-2 mm, a deflectable tip, and a thermistor embedded in a 4-mm distal electrode (EP Technologies, Mountainview, CA, USA). The catheter was inserted into a femoral artery and positioned in the left ventricle using a retrograde aortic approach. The intracardiac electrograms and several electrocardiographic leads were displayed on an oscilloscope and recorded either on paper (Mingograph -7, Siemens-Elema, Solna, Sweden) at a paper speed of $100 \mathrm{~mm} / \mathrm{sec}$, or on optical disk (Labsystem 2.57, C.R. Bard Inc., Billerica, MA, USA). The left ventricular endocardial electrograms were recorded simultaneously at gain settings of 20 and $80 \mathrm{~mm} / \mathrm{mV}$ at filter settings of 50 to $500 \mathrm{~Hz}$. Pacing was performed with a programmable stimulator (Bloom Associates Ltd., Redding, PA, USA). A previously described stimulation protocol using four extrastimuli was performed to induce VT. ${ }^{10}$

During mapping, bipolar electrograms were recorded with the distal pair of electrodes (electrodes 1 and 2) of the mapping catheter. However, when looking for concealed entrainment, to minimize the distance between the pacing and recording sites, electrodes 1 and 3 of the mapping catheter were used for bipolar left ventricular pacing, and electrodes 2 and 4 were used for recording the local endocardial electrogram.
To look for concealed entrainment, pacing was performed with trains of 10 to 15 stimuli at cycle lengths 20 to $150 \mathrm{msec}$ shorter than the VT cycle length. Pacing was performed at all sites at which the local electrogram preceded the QRS complex during VT by at least $70 \mathrm{msec}$, at which there was an isolated diastolic potential, or the local electrogram during VT was abnormal (amplitude $<0.5 \mathrm{mV}$ and duration $>60 \mathrm{msec}^{11}$ ).

\section{Ablation Protocol}

Radiofrequency energy was applied at all sites at which there was concealed entrainment. The radiofrequency energy was delivered as a continuous, unmodulated sine wave at a frequency of $500 \mathrm{kHz}$ (EP Technologies). The power was titrated to maintain an electrode-tissue interface temperature of $60^{\circ} \mathrm{C}$. Once a temperature of $60^{\circ} \mathrm{C}$ was attained, the application of energy was continued for at least 20 seconds. If the VT did not terminate, the energy application was discontinued and mapping was continued. If the VT did terminate, the application was continued for a total of 60 seconds at the final power setting. Programmed ventricular stimulation was then repeated to determine whether the targeted VT was still inducible.

Ablation was considered successful if: (1) the VT was terminated by an application of radiofrequency energy and was no longer inducible by programmed ventricular stimulation, and (2) there were no recurrences of VT during follow-up that had either the same configuration as that of the targeted VT, or the same rate as determined by analysis of stored electrograms in the patients who received an internal cardioverter defibrillator.

\section{Analysis of Local Electrograms}

Radiofrequency energy was delivered at 92 sites, and the endocardial electrograms recorded at each of these sites were analyzed post-hoc. An endocardial potential was considered to be a discrete systolic potential if the potential was discrete from the main component of the ventricular electrogram and completely or partially overlapped with electrical systole, and if the interval between the potential and the next QRS complex (the systolic potential-QRS interval) was equal to the stimulus-QRS interval during concealed entrainment at that site (Fig. 1).

An isolated diastolic potential was defined as 
a discrete potential located entirely within electrical diastole that was separated by an isoelectric segment from the main portion of the ventricular electrogram and that could not be dissociated from the VT by pacing. ${ }^{1}$

\section{Follow-Up}

After the ablation procedure, treatment was continued with the same antiarrhythmic medications at the same dosages that had been used before the ablation procedure. After discharge from the hospital, the patients were seen on an outpatient basis at 2 to 4 month intervals. The mean duration of follow-up was $10 \pm 16$ months (range 2 to 24 months).

\section{Statistical Analysis}

Continuous data are expressed as mean \pm 1 standard deviation. Statistical comparisons were performed using Student's $t$-test, the Fisher exact test, or by Chi-square test, as appropriate. A P value $<0.05$ was considered statistically significant.

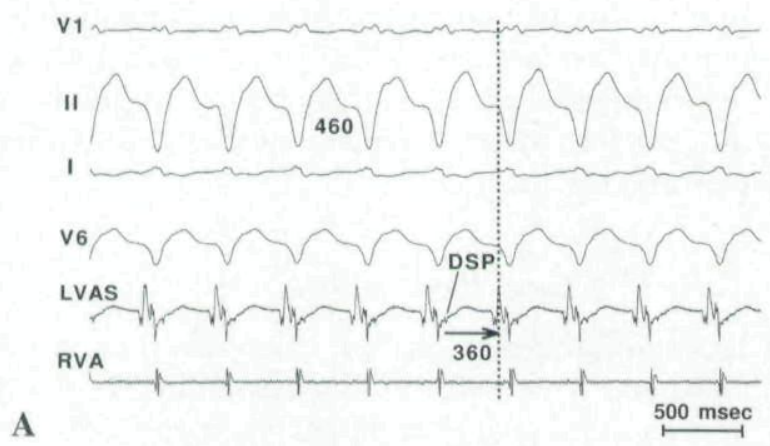

\section{Results}

\section{Prevalence and Characteristics of Discrete Systolic Potentials (Table 1)}

Concealed entrainment was demonstrated at 92 different sites (range 1 to 8 sites for each of the 42 VTs). A discrete systolic potential was present in 7 of the 42 VTs (17\%), at 7 of the 92 sites with concealed entrainment ( $8 \%)$. The mean amplitude of the discrete systolic potentials was $0.06 \pm 0.03 \mathrm{mV}$. The mean duration of the discrete systolic potentials was $46 \pm 35 \mathrm{msec}$. Two of the discrete systolic potentials were located entirely within electrical systole, and five began during electrical systole and extended a mean of $29 \pm 36 \mathrm{msec}$ beyond the QRS complex. In accord with the criteria used to identify discrete systolic potentials, the mean systolic potential-QRS interval $(277 \pm 72 \mathrm{msec})$ did not differ significantly from the mean stimulus-QRS interval (279 $\pm 67 \mathrm{msec})$ during concealed entrainment for the seven VTs in which a discrete systolic potential was identified.

An isolated diastolic potential was present in 19 of the 42 VTs (45\%), at 19 of the 92 sites $(21 \%)$ at which concealed entrainment was demonstrated. The $45 \%$ prevalence of isolated diastolic potentials was significantly greater than the

Figure 1. An example of a discrete systolic potential that occurred entirely within electrical systole. (A) A bipolar recording from the distal pair of electrodes of the mapping catheter at the left ventricular apical septum (LVAS) during ventricular tachycardia (VT) that had a cycle length of $460 \mathrm{msec}$. There is a discrete systolic potential (DSP) that follows the main portion of the ventricular electrogram. The interval between the DSP and the next QRS complex is 360 msec (arrow). Also shown are leads VI, II, I, and V6, and an electrogram recorded at the right ventricular apex (RVA). (B) Concealed entrainment demonstrated by pacing at a cycle length of $380 \mathrm{msec}$ at the same left ventricular septal site where the DSP in Figure $1 \mathrm{~A}$ was recorded. Each of the 12 ECG leads is shown. The asterisk denotes the last entrained QRS complex. As is characteristic of concealed entrainment, the QRS complexes during entrainment and during VT are identical in configuration. The stimulusQRS interval during concealed entrainment is $360 \mathrm{msec}$ (arrow), equal to the interval between the DSP shown in Figure $1 \mathrm{~A}$ and the next QRS complex. $S=$ stimulus. 
TABLE 1

Characteristics of Discrete Systolic Potentials and Isolated

Diastolic Potentials at Sites of Concealed Entrainment in 42 Ventricular Tachycardias

\begin{tabular}{lccc}
\hline & $\begin{array}{c}\text { Discrete } \\
\text { Systolic } \\
\text { Potential }\end{array}$ & $\begin{array}{c}\text { Isolated } \\
\text { Diastolic } \\
\text { Potential }\end{array}$ & $\begin{array}{c}\text { P } \\
\text { Value }\end{array}$ \\
\hline Prevalence & $7 / 42(17 \%)$ & $19 / 42(45 \%)$ & $<0.01$ \\
Amplitude $(\mathrm{mV})$ & $0.06 \pm 0.03$ & $0.06 \pm 0.03$ & 0.9 \\
Duration $(\mathrm{msec})$ & $46 \pm 35$ & $43 \pm 13$ & 0.9 \\
\hline
\end{tabular}

Continuous variables are expressed as mean \pm standard deviation.

$17 \%$ prevalence of discrete systolic potentials $(\mathrm{P}<0.01)$. The characteristics of the isolated diastolic potentials are described in Table 1. There were no significant differences in the amplitude or duration of discrete systolic potentials and isolated diastolic potentials. The interval between the isolated diastolic potential and the next QRS complex was equivalent to the stimulusQRS interval during concealed entrainment at 8 of the 19 sites $(42 \%)$ where an isolated diastolic potential was present.

Both a discrete systolic potential and isolated diastolic potential were present in 6 of the 42 VTs $(14 \%)$, at 6 of the 92 sites $(7 \%)$ where there was concealed entrainment (Fig. 2). At these sites, the mean systolic potential-QRS interval during VT of $263 \pm 67 \mathrm{msec}$ did not differ significantly from the mean stimulus-QRS interval during concealed entrainment of $265 \pm 62$ $\mathrm{msec}$, and both of these values were significantly longer than the mean diastolic potential-QRS interval during VT of $194 \pm 79 \mathrm{msec}(\mathrm{P}<0.01$, Fig. 2).

\section{Results of Catheter Ablation}

Thirty-five of the 42 VTs ( $83 \%$ ) were successfully ablated. The outcome of ablation was successful for each of the seven VTs in which a discrete systolic potential was present at the ablation site $(100 \%)$. Among the 13 VTs in which an isolated diastolic potential, but not a discrete systolic potential, was present at the ablation site, successful ablation was achieved in 11 (85\%). This success rate was not significantly different than the success rate at sites where there was a discrete systolic potential.

\section{Discussion}

\section{Main Findings}

The results of this study demonstrate that a discrete systolic potential may be present at a site of concealed entrainment in close to $20 \%$ of VTs
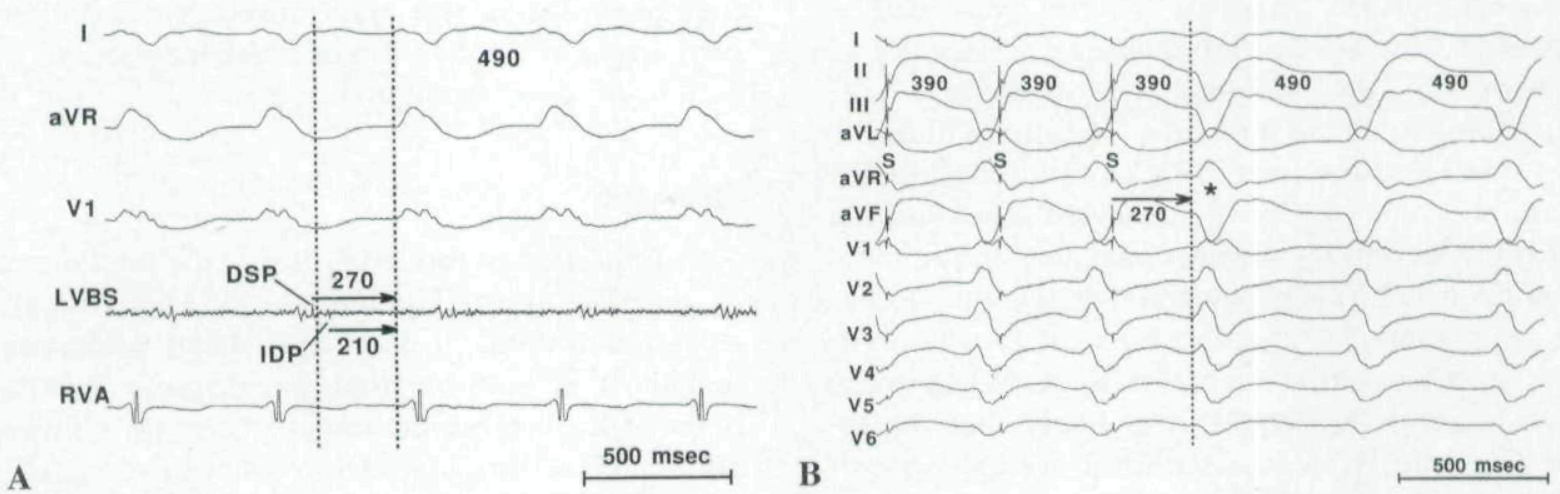

Figure 2. An example of a ventricular tachycardia (VT) in which both a discrete systolic potential (DSP) and an isolated diastolic potential were recorded at the same site. (A) A bipolar recording from the distal pair of electrodes of the mapping catheter at the left ventricular basal septum (LVBS) during VT that had a cycle length of 490 msec. The beginning and end of the diastolic interval are denoted by the dotted lines. A DSP is seen just before the end of electrical systole, and an isolated diastolic potential (IDP) is seen in early diastole. The interval between the DSP and the onset of the next QRS complex is 270 $m s e c$, and the interval between the IDP and the next QRS complex is $210 \mathrm{msec}$. (B) Concealed entrainment demonstrated by pacing at a cycle length of $390 \mathrm{msec}$ at the same left ventricular basal septal site shown in Figure $3 \mathrm{~A}$. Each of the 12 ECG leads is shown. The asterisk denotes the last entrained QRS complex. As expected in concealed entrainment, the QRS complexes during entrainment and during VT are identical in configuration. The stimulus-QRS interval during concealed entrainment is $270 \mathrm{msec}$ (arrow), equal to the interval between the DSP shown in Figure $3 A$ and the next QRS complex. $S=$ stimulus. 
in patients with prior myocardial infarction. Except for the fact that these potentials occur during electrical systole instead of diastole, their characteristics are very similar to those of isolated diastolic potentials. Furthermore, when the interval between the discrete systolic potential and the next QRS complex is equal to the stimulusQRS interval during concealed entrainment, there is a high probability that delivery of radiofrequency at that site will result in successful ablation of the VT, as is the case when the interval between an isolated diastolic potential and the next QRS interval is equal to the stimulus-QRS interval during concealed entrainment. ${ }^{5}$

Isolated diastolic potentials that cannot be dissociated from VT are reliable markers of successful target sites for ablation,,$^{1,3}$ and experimental studies have indicated that they are generated in a zone of slow conduction within the VT reentry circuit. ${ }^{12}$ The results of this study suggest that discrete systolic potentials may also be generated in a zone of slow conduction, indicating that the zone of slow conduction may be penetrated by the VT wavefront simultaneous with global ventricular activation.

\section{Electrogram-QRS Interval and Stimulus-QRS Interval}

In the setting of prior myocardial infarction, diastolic endocardial potentials recorded during concealed entrainment of VT are generated in areas of slow conduction that may or may not be critical for the maintenance of reentry. ${ }^{1,5,8,13}$ Comparison of the interval between the diastolic potential and the next QRS complex during VT with the interval between the stimulus and the next QRS complex during concealed entrainment has been helpful in determining whether a diastolic potential is arising in a critical or noncritical portion of the reentry circuit. When these two intervals are equal, it is likely that the recording site is located within a critical zone of slow conduction and that delivery of radiofrequency energy at that site will be successful in ablating the VT. ${ }^{5,8}$

A similar type of comparison was adapted for use in the present study, and a criterion for the identification of discrete systolic potentials was a systolic potential-QRS interval during VT that was within $10 \mathrm{msec}$ of the stimulus-QRS interval during concealed entrainment. Because radiofrequency catheter ablation of VT was uniformly successful at the sites where these systolic po- tentials were recorded, it is likely that the sites were in fact within a critical portion of the reentry circuit.

\section{Systolic and Diastolic Potentials Recorded at the Same Site}

It is especially important to examine the systolic interval for the presence of a discrete systolic potential when there is an isolated diastolic potential recorded at a site where the diastolic potential-QRS interval during VT is shorter than the stimulus-QRS interval during concealed entrainment. In the present study, both a discrete systolic potential and an isolated diastolic potential were recorded at the same endocardial site in almost $20 \%$ of VTs. In each of these cases, it was the systolic potential-QRS interval, not the diastolic potential-QRS interval, that equaled the stimulus-QRS interval. Had the presence of the discrete systolic potential not been recognized, it might have been incorrectly concluded that these sites were unlikely to be effective target sites for ablation.

Based on the findings of this study, it seems likely that discrete potentials generated in a critical zone of slow conduction may be either systolic or diastolic, depending on the relative timing between depolarization of this zone by the reentrant wavefront and global ventricular depolarization. The presence of both types of potentials at the same site may represent discontinuous, sequential activation of adjacent portions of a zone of slow conduction.

\section{Limitations}

A limitation of this study is that the prevalence of discrete systolic potentials at sites of concealed entrainment may have been underestimated. It is possible that the discrete systolic potentials were buried within the main component of the ventricular electrogram in some VTs and therefore could not be seen. Bipolar recordings with electrodes that were smaller and more closely spaced than the electrodes used in the present study may have allowed a greater degree of precision in identifying discrete systolic potentials.

\section{Conclusions}

The value of diastolic potentials for identifying target sites for ablation of VT in patients with 
prior myocardial infarction has been well recognized, ${ }^{1-9}$ but there has been no mention in prior studies of the possible importance of potentials recorded during systole. This study demonstrates the importance of scrutinizing systole for the presence of discrete potentials during mapping procedures, because discrete systolic potentials also may be a reliable indicator of a successful target site for ablation of VT.

\section{References}

1. Fitzgerald DM, Friday KJ, Yeung-Lai-Wah JA, et al: Electrogram patterns predicting successful catheter ablation of ventricular tachycardia. Circulation 1988;77: 806-814.

2. Kuck KH, Schluter M, Geiger M, et al: Successful catheter ablation of human ventricular tachycardia with radiofrequency current guided by an endocardial map of the area of slow conduction. PACE 1991;14:10601071.

3. Fitzgerald DM, Friday KJ, Yeung-Lai-Wah JA, et al: Myocardial regions of slow conduction participating in the reentrant circuit of multiple ventricular tachycardias: Report on ten patients. J Cardiovasc Electrophysiol 1991;2:193-206.

4. Morady F, Harvey M, Kalbfleisch SJ, et al: Radiofrequency catheter ablation of ventricular tachycardia in patients with coronary artery disease. Circulation 1993; 87:363-372.

5. Stevenson WG, Khan H, Sager P, et al: Identification of reentry circuit sites during mapping and radiofrequency ablation of ventricular tachycardia late after myocardial infarction. Circulation 1993;88:1647-1670.
6. Gonska BD, Cao K, Schaumann A, et al: Catheter ablation of ventricular tachycardia in 136 patients with coronary artery disease: Results and long-term followup. J Am Coll Cardiol 1994;24:1506-1514.

7. Wilber DJ, Kopp DE, Glascock DN, et al: Catheter ablation of the mitral isthmus for ventricular tachycardia associated with inferior infarction. Circulation 1995:92:3481-3489.

8. Bogun F, Bahu M, Knight B, et al: Comparison of effective and ineffective target sites that demonstrate concealed entrainment in patients with coronary artery disease undergoing radiofrequency ablation of ventricular tachycardia. Circulation 1997; 95:183190.

9. Bogun F, Bahu M, Knight B, et al: Response to pacing at sites of isolated diastolic potentials during ventricular tachycardia in patients with previous myocardial infarction. J Am Coll Cardiol 1997;30:505-513.

10. Hummel J, Strickberger S, Daoud E, et al: Results and efficiency of programmed ventricular stimulation with four extrastimuli compared with one, two, and three extrastimuli. Circulation 1994;90:2827-2832.

11. Cassidy DM, Vassallo JA, Miller JM, et al: Endocardial catheter mapping in patients in sinus rhythm: Relationship to underlying heart disease and ventricular arrhythmias. Circulation 1986;73:645-652.

12. El-Sherif N, Mehra R, Gough WB, et al: Reentrant ventricular arrhythmias in the late myocardial infarction period. Interruption of reentrant circuits by cryothermal techniques. Circulation 1983;68:644-656.

13. Stevenson WG, Friedman PL, Ganz LI: Radiofrequency catheter ablation of ventricular tachycardia late after myocardial infarction. J Cardiovasc Electrophysiol 1997;8:1309-1319. 
This document is a scanned copy of a printed document. No warranty is given about the accuracy of the copy. Users should refer to the original published version of the material. 